\title{
The silicon detector of the muon g-2 experiment at JPARC
}

\section{Tsutomu Mibe*i}

Institute of Particle and Nuclear Studies, KEK, Japan

E-mail: mibedpost.kek.ip

\begin{abstract}
A new measurement of the anomalous magnetic moment $(g-2)$ and electric dipole moment $(\mathrm{EDM})$ of the positive muon is proposed with a silicon tracking detector to detect positrons from muon decay, together with a technique utilizing an ultra-cold muons accelerated to $300 \mathrm{MeV} / \mathrm{c}$ and a $66 \mathrm{~cm}$-diameter compact muon storage ring without focusing-electric field. Design strategy and requirements for the tracking detector with double-sided silicon strip sensors and evaluation of the sensor are discussed.
\end{abstract}

The 20th Anniversary International Workshop on Vertex Detectors - VERTEX 2011

June 19 - 24, 2011

Rust, Lake Neusiedl, Austria

\footnotetext{
* Speaker.

${ }^{\dagger}$ work in collaboration with T. Kakurai, T. Kohriki, N. Saito, O. Sasaki, K. Ueno , and the J-PARC muon g-2/EDM collaboration
} 


\section{Introduction}

The anomalous magnetic moment of the muon $\left(a_{\mu}=(g-2) / 2\right)$ is directly sensitive to the electromagnetic, strong, weak forces, as well as possible extensions of the Standard model [四. The present experimental value for the muon $g-2$ is from the E821 experiment at BNL, which achieved a sensitivity of $0.54 \mathrm{ppm}$ []. This measured value, $a_{\mu}=0.00116592080$ (54) (33), deviates from the calculated value by 3 standard deviations [ [3]. The success of the E821 experiment stimulated significant progress of $e^{+} e^{-}$experiments and related theories. On the other hand, the precision measurement of $a_{\mu}$ is only available from the E821. The continuation of the E821-type experiment at FNAL E989 [四] is proposed and is under preparation. We take an yet another approach to measure this fundamental quantity with a completely new experimental technique, thus providing a completely independent measurement.

\section{Measurements of muon dipole moments with ultra-cold muon beam}

The spin orientation of the longitudinally polarized muon follows its momentum direction when it is stored in the static electro-magnetic field, $\vec{B}$ and $\vec{E}$. The anomalous magnetic moment $a_{\mu}$ introduces an additional precession with its precession vector $\vec{\omega}_{a}$ given by

$$
\vec{\omega}_{a}=-\frac{e}{m_{\mu}}\left[a_{\mu} \vec{B}-\left(a_{\mu}-\frac{1}{\gamma^{2}-1}\right) \frac{\vec{\beta} \times \vec{E}}{c}\right] .
$$

Since $a_{\mu} \sim \alpha /(2 \pi) \sim 0.00116$, a choice of magic energy, $\gamma=29.4$ would reduce the above formula to a simple form,

$$
\vec{\omega}_{a}=-\frac{e}{m_{\mu}} a_{\mu} \vec{B}
$$

Therefore, a precision measurement of $\vec{\omega}_{a}$ in precision field $\vec{B}$ will realize precise determination of $a_{\mu}$. This approach was taken in the BNL E821 experiment.

While running at the magic energy is advantageous to simplify the spin equation of motion, there is another approach to avoid the complication of Eq. 2. , i.e. elimination of the electric field. We propose to measure the muon $g-2$ without the focusing electric field by employing an ultracold muon beam, where the transverse momentum dispersion, $\sigma\left(p_{T}\right)$ is significantly smaller than its longitudinal momentum, $p_{L}$.

Such a beam can circulate in the storage ring without, or ultra-weak if any, the focusing field for the duration of the measurements. Elimination of the electric field would greatly simplify the precession frequency as Eq. 2.2, or it can be written as follows by introducing an explicit contribution from the electric dipole moment

$$
\vec{\omega}_{a}+\vec{\omega}_{\eta}=-\frac{e}{m_{\mu}}\left[a_{\mu} \vec{B}-\frac{\eta}{2}(\vec{\beta} \times \vec{B})\right],
$$

where $\eta$ is the electric dipole moment (EDM), and $\omega_{\eta}$ is its precession vector due to the EDM. Since the rotation axes due to $a_{\mu}$ and $\eta$ are orthogonal, separation of these signals is possible.

We consider the case of

$$
\frac{\sigma\left(p_{T}\right)}{p_{L}} \sim 10^{-5}
$$




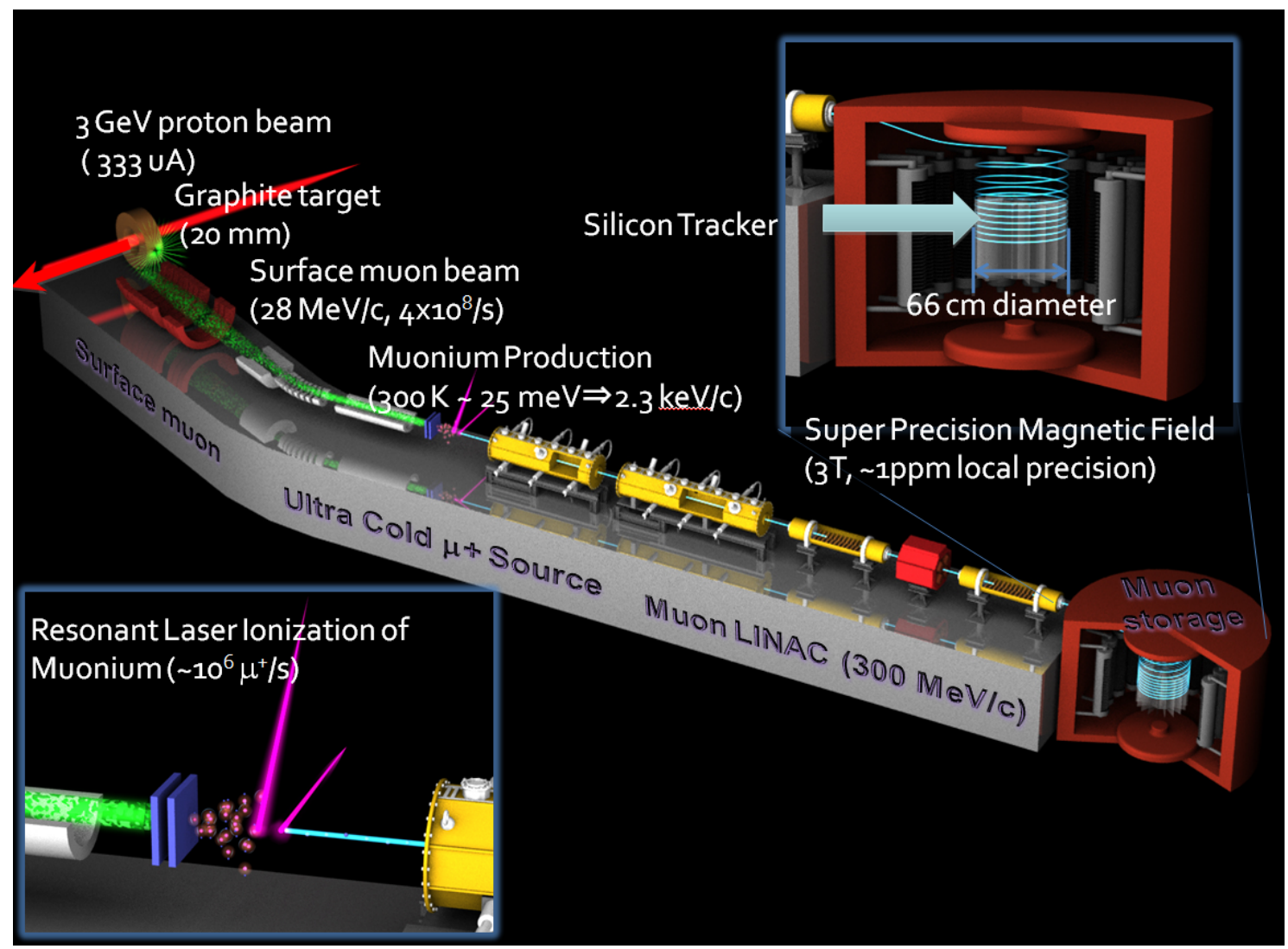

Figure 1: The J-PARC $g-2$ experiment

with the $B=3 \mathrm{~T}$ and $p=300 \mathrm{MeV} / c$, which corresponds to the radius of cyclotron motion of $33.3 \mathrm{~cm}$. The $g-2$ precession is measured for five times its dilated lifetime of muon $(5 \times 6.6 \mu \mathrm{sec})$. This corresponds to travel length of $8 \mathrm{~km}$, or about 4,000 turns. The beam spread due to the transverse momentum dispersion in Eq. 2.4 is $80 \mathrm{~mm}$ after 4,000 turns, which can easily be kept in the storage field.

We plan to realize such an experiment at J-PARC [8]. The overview of the J-PARC g-2 experiment is shown in Fig $\mathbb{~ W . ~ T h e ~ g o a l ~ f o r ~ t h i s ~ n e w ~ e x p e r i m e n t ~ i s ~ t o ~ r e a c h ~ t h e ~ s e n s i t i v i t y ~ o f ~} a_{\mu}$ down to $0.1 \mathrm{ppm}$ level.

\subsection{Ultra-Cold Muon Beam}

The ultra-cold muons will be produced in the following sequence. At the J-PARC [ $[8]$, the $3 \mathrm{GeV}$ proton beam, running at $25 \mathrm{~Hz}$, will hit the Muon graphite target in the M2 tunnel at Material and Life Science Facility (MLF), and produce pions which will be stopped in the target. Those $\pi^{+}$that stop on the surface of the target decay to $4 \mathrm{MeV} \mu^{+}$, which will be collected using a large-aperture capture solenoid, and followed by transport magnets, to a Mu production target. The transported surface muons will stop in the $\mathrm{Mu}$ production target, and form $\mathrm{Mu}\left(\mu^{+}\right.$-electron atoms). The Mu behaves like hydrogen atoms and diffuses from the target. It is important that this target be at room temperature $(300 \mathrm{~K} \sim 25 \mathrm{meV})$ so that the $\mathrm{Mu}$ atoms drift slowly. Pulsed lasers with $122 \mathrm{~nm}$ (Lyman- $\alpha$ ) and $355 \mathrm{~nm}$ wavelength will ionize the Mu at around the target surface, 
producing ultra-cold polarized muons. The muons are then accelerated by muon linac to reach the energy of $320 \mathrm{MeV}$. The expected transverse dispersion of this beam is $10^{-5}$. The beam intensity is $4 \times 10^{4} /$ pulse.

\subsection{Muon Injection and Storage}

The ultra-cold muon beam will be injected to the precision magnetic storage ring which consists of a $3 \mathrm{~T}$ solenoid with $66 \mathrm{~cm}$-diameter, a anti-Helmholtz type kicker, and a magnetic field monitor system. The magnetic field shape of the solenoid is designed so that the muon beam is injected into the storage ring from the upper side of the solenoid magnet by making a vertical spiral trajectory. Spirally-falling muons are stopped in the storage region by the anti-Helmholtz type kicker with $150 \mathrm{~ns}$ pulse full width (half-sine) and 1.3 Gauss. The magnetic field in the storage region is carefully shimmed by the correction coils to achieve the field uniformity of $0.1 \mathrm{ppm}$ level. The absolute magnitude of the magnetic field is periodically mapped by the NMR proving system. The magnetic field strength and distribution in the muon storage region are stationary monitored by Hall-effect sensors which are installed in the vicinity of the muon storage region.

\subsection{Detection of decay positrons}

After injection into the muon storage region, the positive muons circulate in a plane parallel to the magnetic field with a radius of $33 \mathrm{~cm}$. They decay into a positron and two neutrinos with a time-dilated lifetime of $6.6 \mu \mathrm{s}$. The anomalous muon precession period is $2.2 \mu \mathrm{s}$, which is about 300 times the cyclotron period ( 7.4 ns), i.e. the muon spin rotates 360 degrees in every 300 turns in the storage region. Because of parity violation in the weak decay of the muon, there is a correlation between the muon spin and the direction of the positron momentum. The correlation (analyzing power, $A$ ) has an energy and angular dependence.

In the muon center of mass frame, the positrons tend to decay towards the direction of the muon spin at the maximum positron energy of $E_{\max }=52.8 \mathrm{MeV}$ (the Michel edge). The analyzing power becomes smaller in the mid-energy range of $E / E_{\max } \sim 0.5$, then becomes negative in the lowest energy range of $E / E_{\max } \sim 0$, where the direction of the positron tends to be opposite to the muon spin direction. This correlation allows one to measure the time dependence of the muon spin direction by detecting positrons and their energy as a function of time.

This correlation remains in the laboratory frame. Higher analyzing power is obtained with the higher energy threshold $\left(E^{t h}\right)$, while the number of positrons decreases. The statistical uncertainty is proportional to the figure-of-merit (FOM) $C^{\text {th }} A^{2}$ as shown in Fig. Z, where $C^{\text {th }}$ is number of positrons above the energy threshold of $E^{\text {th }}$. The FOM becomes the maximum at around $E^{\text {th }}=$ $190 \mathrm{MeV}$. The maximum positron energy in the laboratory frame is $310 \mathrm{MeV}$. Thus the positron detector should be efficient, especially to detect positrons in the energy region above $\sim 190 \mathrm{MeV}$.

Due to the Lorentz boost, the positron direction is confined to a narrow cone parallel to the parent muon direction. The positrons curl into the inner part of the muon storage region where the magnetic field is as strong as that in the muon storage region $(3 \mathrm{~T})$. The positron momentum component parallel to the magnetic field causes it to drift vertically. The detector system needs to accept positrons which curl to the inside of the muon storage region with a vertical drift. Monte Calro study showed a detector system of a tube-shaped sensitive-volume inside the muon storage area with $40 \mathrm{~cm}$ in height and $27 \mathrm{~cm}$ in radius is sufficiently large. 


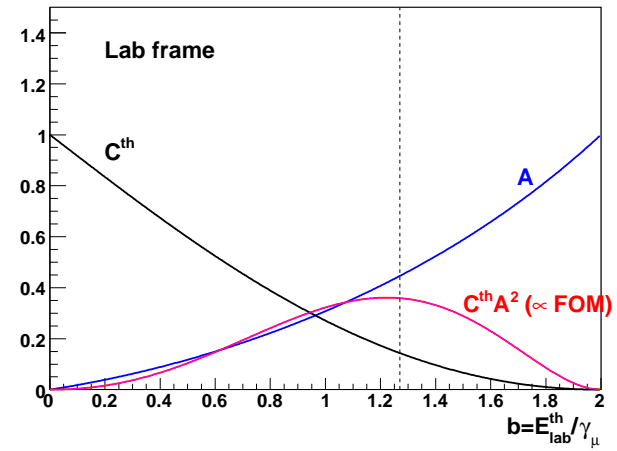

Figure 2: Figure of merit vs threshold on minimum positron energy

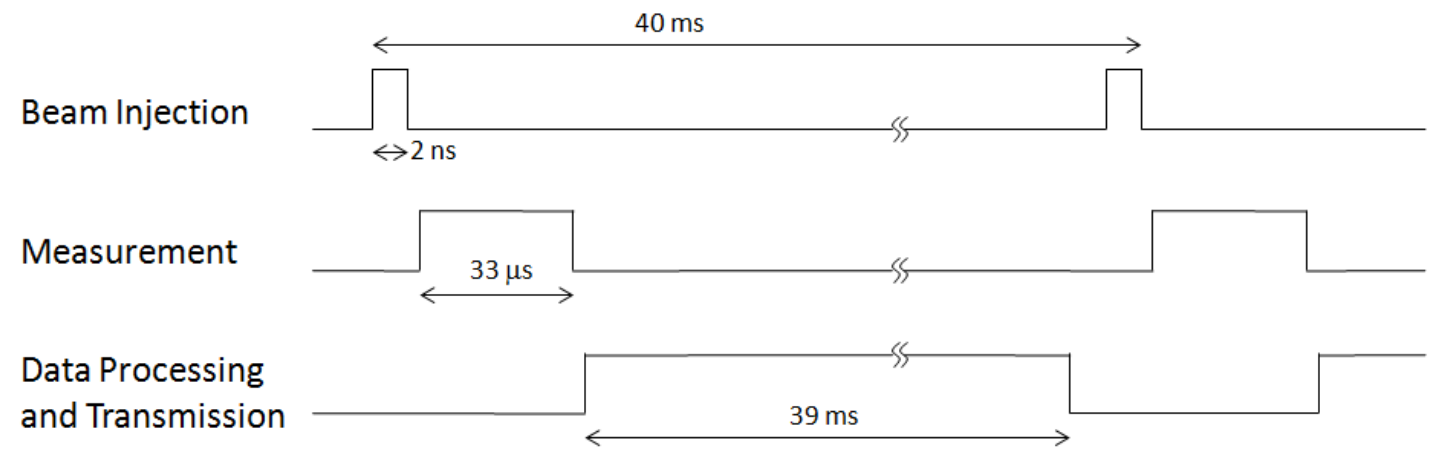

Figure 3: Time sequence of the $g-2$ precession measurement

The time sequence of the $g-2$ measurement is shown in Fig. 13. The muon beam spill comes every $40 \mathrm{~ms}$ synchronously with the J-PARC RF signal. The proposed rate of the muon beam is $10^{6} / \mathrm{s}=40000$ muons per spill. About 10 percent of the decay positrons, 4000 positrons per spill, survive the minimum energy cut mentioned above. We anticipate that the contamination in the detector from positrons with other energies would be of the same order as the signal positrons. Therefore, about 10000 positrons enter the detector system per spill. The instantaneous rate of the whole detector system will be 10000 muons $/ 6.6 \mu \mathrm{s}=1.6 \mathrm{GHz}$ at injection. This becomes $10 \mathrm{MHz}$ after $33 \mu \mathrm{s}$. The individual detector rate would be this rate times the number of detector hits per track divided by the number of channels. The individual detectors must keep their performance (resolution, efficiency, response function) stable as a function of time in order not to introduce any undesired bias in the timing distribution which in turn will be a source of systematic uncertainty.

The detectors and their readout electronics must operate in the $3 \mathrm{~T}$ magnetic field. Any electric field from the detector and readout electronics should be sufficiently small so that the electric field in the muon storage region is negligible for the measurement (a few $\mathrm{mV} / \mathrm{m}$ for a ppb systematic uncertainty). 


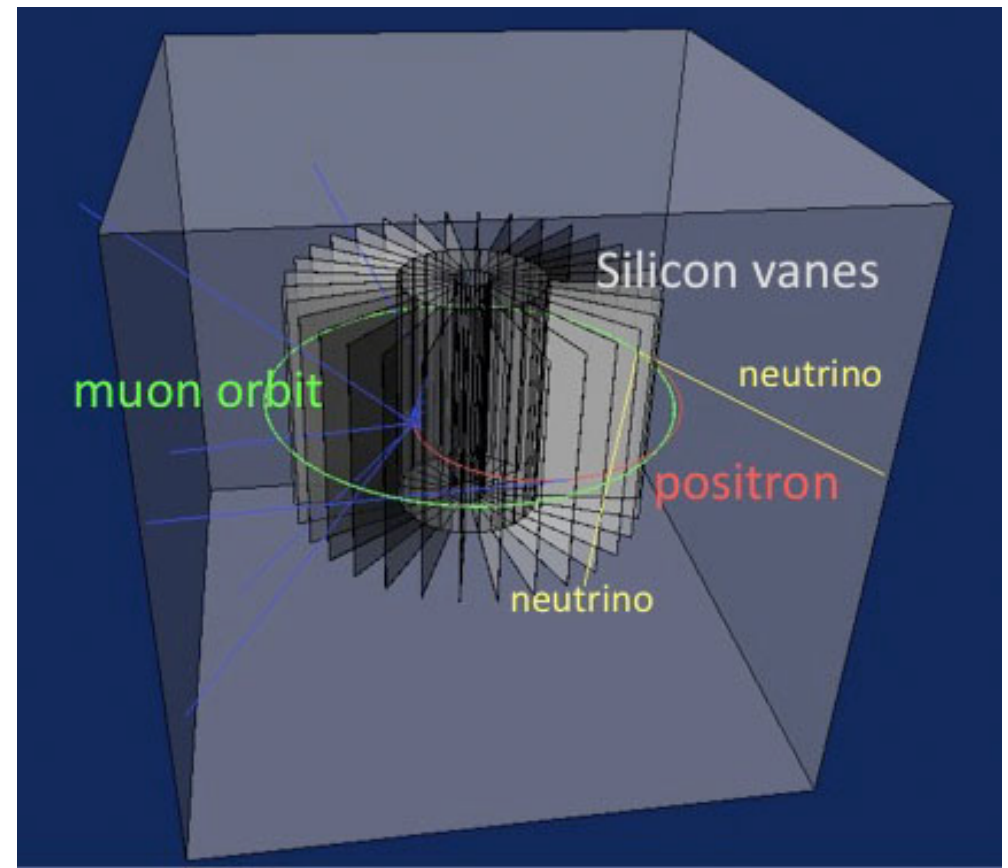

Figure 4: Layout of the positron detector system. The muon storage orbit is represented as a green curve. A muon decay is simulated by a Monte Calro simulation with GEANT4. A positron and neutrinos from the muon decay are shown by red and yellow curves, respectively. Blue lines represent gamma rays due to passage of a positron through detector material.

\section{Silicon tracker}

An array of radial vanes made up of silicon-strip sensors is considered as a positron-tracking detector as shown in Fig. 因. As discussed in the previous section, instantaneous rate will be as high as $1.6 \mathrm{GHz}$ in the detection volume. High granularity is desired to suppress systematic uncertainty from pile-up. Tracking the positrons with position sensitive detectors is beneficial in a sense that effects of pile-up can progressively be suppressed by requiring hit-to-hit correlations in the track reconstruction. The existence of the strong magnetic field in the detection volume provides for momentum analysis.

Silicon strip detectors are considered for the tracking detector since these detectors are compact, highly segmented, have stable gain, and do not introduce high electric field in the detection volume. The sensitive area of the detector is made up with double-sided AC-coupled rectangular silicon sensors with radial and axial strips with $200 \mu \mathrm{m}$ pitch. A vane consists of 8 silicon strip sensors which are placed in a $4 \times 2$ matrix. In a vane, strip hits from each sensor are read out individually. The size of each sensor is $6 \mathrm{~cm}$ wide and $12 \mathrm{~cm}$ high. A total of 98 units of silicon strip planes will be radially placed in the detection volume to efficiently detect the circular tracks of the positrons. 48 units are used to form a set of vanes in the upper half of the detection volume, while another 48 units are for the lower half. There are 691k strips in total. Figure 5 shows a conceptual mechanical design of the silicon vane unit. It consists of eight silicon sensors supported by a L-shaped PCB board. Front-end ASICs are located in both sides of the PCB board.

Figure. 6 shows examples of positron trajectories with three different energies. Here image of 


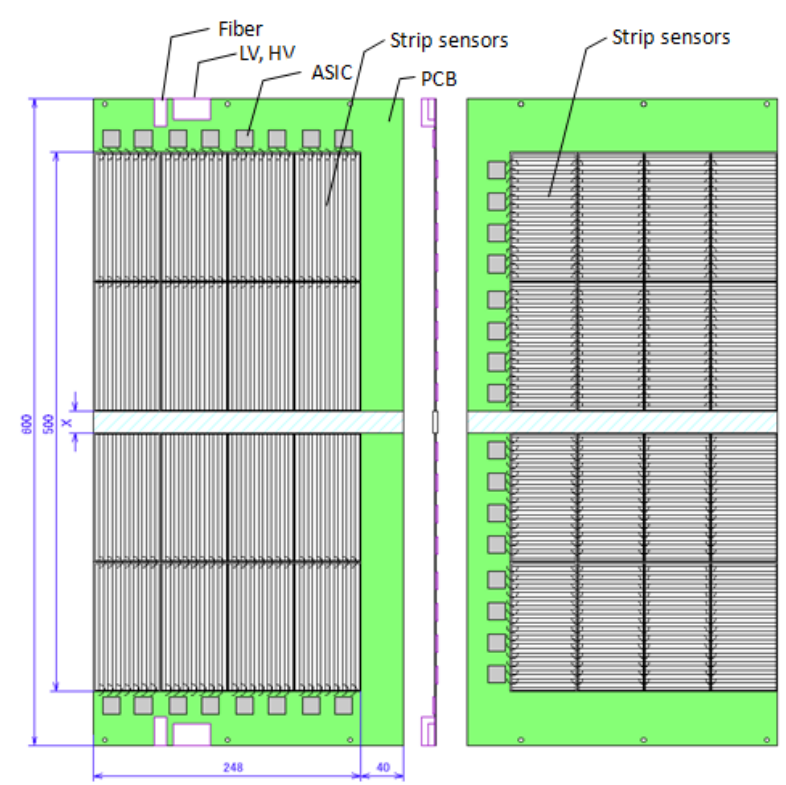

Figure 5: A conceptual mechanical design of the silicon strip vane with double-sided silicon strip sensors (side view).

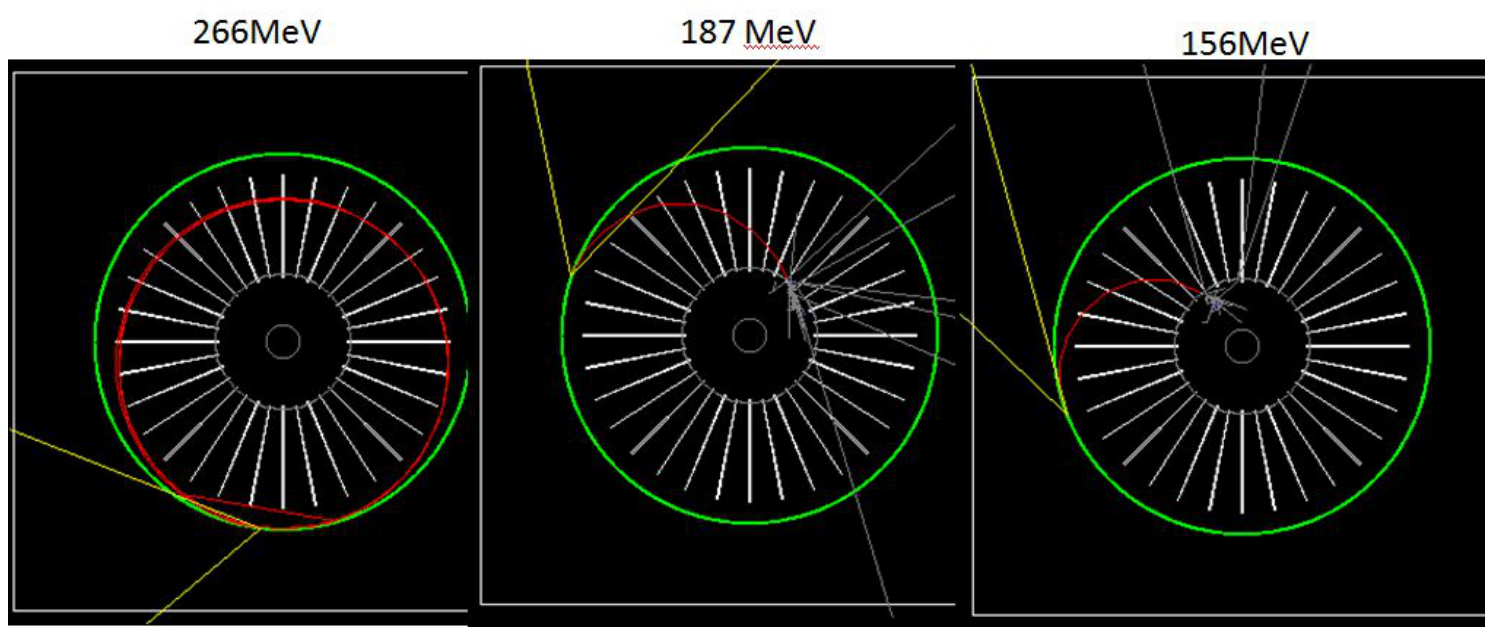

Figure 6: Example positron trajectories in the detector system at three different energies of positrons. The green circle is the muon beam orbit. The red trajectory is the trace of the positron track. The white tracks are photons.

silicon tracking plane was shown as white radial vanes. It can be seen that at the higher positron energy, the track path-length in the detection volume becomes longer, and thus more hits are registered in the tracker. On the other hand, acceptance becomes less and less at lower energies. At energies below $50 \mathrm{MeV}$, the tracker is completely insensitive to these unwanted positrons, i.e. the radius of curl-up is so small that it can not be seen by any vane. Note that we are not sensitive to positrons at the highest energy $(\mathrm{E}>280 \mathrm{MeV})$ as well since the radius of the positron track is similar to that of muons.

A double-sided silicon strip sensor is considered as a sensor for the silicon tracker. Hamamatsu 
photonics co. (HPK) supplies a double-sided strip sensor from a 6 inch wafer with dimension of $12 \mathrm{~cm} \times 6 \mathrm{~cm}$ and $0.32 \mathrm{~mm}$ in thickness, which has been developed for the Belle-II SVD [12]]. Our current design assumes that similar size of sensor with $0.2 \mathrm{~mm}$ strip pitch, which can be available with minor modifications from the Belle-II DSSD sensor. The sensor is made of n-type bulk substrate with implantation of $\mathrm{p}^{+}$in the $\mathrm{p}$-side and $\mathrm{n}^{+}$in the $\mathrm{n}$-side of the sensor plane. Orientation of strips in the p-side is orthogonal to that in the n-side. The depletion voltage of the sensor is about $80 \mathrm{~V}$. The energy needed for creation of an electron-hole pair in the sensor is $3.6 \mathrm{eV}$. Total number of electron-hole pairs created by the minimum ionization particle in a silicon sensor of $0.32 \mathrm{~mm}$ thickness is about $25 \mathrm{k}$ pairs. Signal is read out by an AC coupling capacitor made of a thin silicon dioxide film implanted on the strips. The capacitance of the strip depends on its length and width, which needs to be determined by requirements to be studied by Monte Carlo simulation. The intrinsic timing resolution of the silicon sensor is about $3 \mathrm{nsec}$ estimated from the drift velocity $(0.1 \mathrm{~mm} / \mathrm{nsec})$.

Test modules with a fast preamplifier-shaper-discriminator chip (ASD) [[13] were constructed to evaluate the performance of DSSD sensors with an emphasis on timing responses. This ASD was not originally designed for silicon strip readout, but it has a novel characteristics of fast rise-time (10 nsec) and shaping-time ( $25 \mathrm{nsec})$. The ASD consists of 16 channels of preamplifier, shaper, and comparator with binary outputs as well as analog monitor outputs for each channel. We have been evaluating timing performance and its rate dependence with a short-pulsed diode laser. The diode laser produces a laser pulse of $1060 \mathrm{~nm}$ with a pulse width of $55 \mathrm{ps}$ (FWHM) at repetition rate of upto $80 \mathrm{MHz}$. A test module was set on a support structure with a XY moving stage which enable it to adjust relative position between laser spot and the test module with a micro-meter accuracy. The measured the laser spot size was $120 \mu m(\sigma)$ with a multi-mode optical fiber and $60 \mu m$ with a single-mode optical fiber. The pulse amplitude of $35 \mathrm{mV}$ was observed for laser power of $25 \mathrm{nW}$ which is equivalent to $25 \mathrm{k}$ electron-hole pairs in the sensor. Studies on rate dependence at various repetition rates of the laser is on going.

Other parts of $R \& D$ are on electric field and magnetic field induced in the vicinity of the silicon sensor. In order to realize electric field free environment to the level of $0.1 \mathrm{ppm}$ precision of $a_{\mu}$, the electric field strength in the muon storage region must be much smaller than $10^{-2} \mathrm{~V} / \mathrm{cm}$. The stray electric from the sensor has been studied by a finite element calculation tool for the 2D static electric problem (POISSON). The calculation showed that Majority of electric field lines $(3 \mathrm{kV} / \mathrm{cm})$ are confined in the silicon sensor when the sensor is fully depleted. A tiny but nonnegligible fraction of electric field line of the order of $1 \mathrm{~V} / \mathrm{cm}$ at the muon storage region is leaked from a potential gap at the outer side of the guard ring on the sensor. This requires to introduce an electric shield between the detector system and the muon storage region. A care must be taken for such shield not to introduce eddy current on the shield material due to the kicker field.

Detector components should not disturb the magnetic field in the muon storage region. Metal elements and many of non-metal material have non-zero magnetic susceptibility [14]]. They induce additional magnetic field when they are placed under a magnetic field. A careful design of the detector structure is necessary to minimize use of ferro-magnetic material. In many cases, information on the material used in the detector components is not disclosed, especially for the integrated-circuit components, such as FPGA, optical transceiver, etc. The effective magnetic susceptibility of those components was measured with a NMR probe under the $1.6 \mathrm{~T}$ superconducting 
solenoid magnet in KEK to test if these components meet the requirement.

A GEANT-based simulation model was developed to evaluate the tracking performance. A track-finding algorithm based on the Hough transformation is being studied in the pattern recognition of the hits in the tracker [피].

\section{Summary}

An experiment to measure the anomalous magnetic moment of the positive muon $a_{\mu}$ down to the level of $0.1 \mathrm{ppm}$ is proposed at J-PARC with high-rate positron tracking detector in a $66 \mathrm{~cm}$ diameter precision magnetic storage ring.

The time and momentum of positrons from muon decay is measured by the positron tracking detector. The conceptual design of the detector is developed by utilizing double-sided silicon-strip sensors, which is anticipated to meet requirements of stability against rate with enough granularity, operation under the $3 \mathrm{~T}$ magnetic field, and minimal leakage electric field as well as minimum distortion of magnetic field. R\&D studies on the DSSD sensor with emphasis on timing responses, rate stability, and impact on electric/magnetic field in the vicinity of the DSSD sensor are in progress.

\section{References}

[1] J.P. Miller, et al., Rep. Prog. Phys. 70, 795 (2007)

[2] G.W. Bennett et al., Phys. Rev. Lett 92, 161802 (2004), G.W. Bennett et al., Phys. Rev. D 73, 072003 (2006).

[3] K. Hagiwara, R. Liao, A. D. Martin, D. Nomura and T. Teubner, J. Phys. G38, 085003 (2011), M. Davier, A. Hoecker, B. Malaescu and Z. Zhang, Eur. Phys. J. C71, 1515 (2011)

[4] B. L. Roberts, Nucl. Phys. Proc. Suppl. 218, 237 (2011).

[5] G. Charpak, et al, Phys. Lett. 1, 16 (1962)

[6] J. Bailey, et al, Nuovo Cimento A 9, 369 (1972)

[7] J. Bailey, et al, Nucl. Phys. B 150, 1 (1979)

[8] http:j-parc.jp/index-e.html

[9] K. Nagamine, et al., Phys. Rev. Lett. 74, 4811 (1995).

[10] P. Bakule, et al., Nucl. Instrum. Meth. B. 266, 335 (2008).

[11] The J-PARC g-2 collaboration, proposal submitted to J-PARC PAC (P34), 2009

[12] M. Friedl, et al., in these proceedings

[13] O. Sasaki, et al. (ATLAS TGC Collaboration), http://atlas.kek.jp/tgcelec/ASD/ASD-PRR.pdf

[14] Handbook of Chemistry and Physics 89th edition, CRC press (2008)

[15] K. Ueno, the 2011 IEEE Nuclear Science Symposium, Medical Imaging Conference, in preparation 